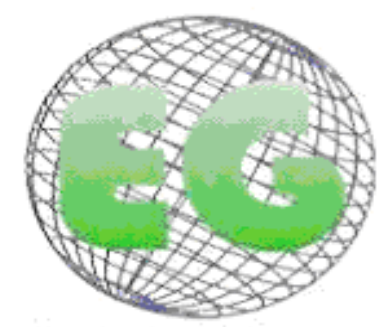

ISSN 1695-6141 N'25.

www.um.es/egloball

\title{
REVISIONES
}

\section{Sistematizando la literatura sobre acreditación hospitalaria: de 2005 a 2010}

Sistematizando a literatura sobre acreditação hospitalar: de 2005 a 2010

\section{*Da Motta Duarte, MS., **Silvino, ZR.}

*Enfermeira. Mestranda em Ciências do Cuidado em Saúde da Escola de Enfermagem Aurora de Afonso Costa da Universidade Federal Fluminense. Gestora do Serviço de Enfermagem do Hospital da Polícia Militar de Niterói-RJ. ** Doutora em Enfermagem. Professora Titular de Administração em Enfermagem e do Mestrado Acadêmico em Ciências do Cuidado em Saúde da Universidade Federal Fluminense. Brasil.

Palabras clave: acreditación; calidad de asistencia en salud; acceso y evaluación; enfermería.

Palavras-chave: acreditação; qualidade da assistência à saúde; acesso e avaliação; enfermagem

Keywords: accreditation; health care quality; access, and evaluation; nursing

\section{RESUMEN}

Objetivo: Ver cómo el tema de acreditación y / o evaluación de los servicios de salud está siendo tratado en la literatura.

Método: una revisión integradora de la literatura en bases de datos electrónicas: LILACS, IBECS, BDENF, y MEDLINE. Los descriptores utilizados fueron: Acreditación, Calidad de la atención de salud, acceso y evaluación, con una ventana de tiempo entre los años 2005 a 2010.

Resultados: el estudio incluyó a 18 (dieciocho) artículos completos en línea y una disertación. Para analizar los datos, se utilizó la lectura interpretativa y el análisis temático. Surgieron categorías: Criterios y Proceso de Evaluación de Resultados, beneficios de asistencia y cambio cultural.

Conclusión: la acreditación de los servicios de salud es una herramienta que se utiliza en todo el mundo y ha evolucionado continuamente sus procesos para hacer frente a la excelencia para alcanzar sus metas. A pesar de no evitar la aparición de malas prácticas, ha sido una oportunidad de las instituciones de salud para mejorar la calidad de la atención, la atención y cuidado a los pacientes. En lo que respecta al Departamento de Enfermería, el estudio identificó su participación en el proceso, haciendo hincapié en la necesidad de ajustes para una evaluación más efectiva de la prestación de la atención y la conciencia de todo el equipo.

\section{RESUMO}

Objetivo: verificar como a temática acreditação e/ou avaliação dos serviços de saúde está sendo abordada na literatura. 
Método: revisão integrativa da literatura nas bases de dados eletrônicas: LILACS, IBECS, BEDENF, e MEDLINE. Foram utilizados os descritores: Acreditação, Qualidade da assistência à saúde, acesso e avaliação, com recorte temporal entre os anos de 2005 a 2010.

Resultados: foram analisados 18(dezoito) artigos na íntegra online e uma dissertação de mestrado. Para analisar os dados encontrados, utilizamos a leitura interpretativa e análise temática. Emergiram as categorias: Critérios de resultado e processo de avaliação; Benefícios para a assistência e Mudança cultural.

Conclusão: a acreditação dos serviços de saúde é uma ferramenta que está sendo utilizada mundialmente e tem evoluído seus processos continuamente para dar conta de alcançar com excelência seus objetivos. Apesar de não evitar a ocorrência de erros profissionais, tem sido uma oportunidade das instituições de saúde melhorarem a qualidade do atendimento, atenção e cuidado ao paciente. No que se refere ao Serviço de Enfermagem, o estudo possibilitou identificar a sua participação no processo, pontuando a necessidade de ajustes para avaliação mais efetiva da prestação do cuidado e conscientização de toda equipe.

\section{ABSTRACT}

Objective: to verify how the issuetheme of accreditation and / or assessmentevaluation of health care services is treated being discussed in the literature.

Method: integrative review of the literature in electronic databases: LILACS, IBECS, BEDENF, and MEDLINE. Descriptors used were used: accreditation, health care quality, access and evaluation, with a temporal cut between the years 2005 to 2010 .

Results: were examined 18 (eighteen) full articles online and a master dissertation were examined. To analyze our data, we used the interpretive reading and thematic analysis. Categories emerged: Outcome and evaluation process criteria; Benefits for care and Cultural change.

Conclusion: accreditation of health care services is a tool being used globally and has continually developed itstheir processes to to account for reach its their goals with excellence. Although not preventing the occurrence of professional errors, it has been an opportunity forof health care institutions to improve the quality of assistance, attention and care to patients. Concerning to the Nursing Department, the study identified itstheir participation in the process, stressing the need for adjustments for more effective evaluation of care provision and awareness of the whole team.

\section{INTRODUCCIÓN}

En la medida en que se suceden cambios en las ciencias de la salud, en los acontecimientos mundiales, en las formas educativas y en las condiciones sociales, afectadas por las tendencias políticas y económicas actuales, ha sido un desafío asegurar la calidad en los servicios de salud. Estudios recientes relatan que la gestión de la calidad ofrece una opción para la reorientación gerencial de las organizaciones. ${ }^{(1)}$ Las nuevas tendencias en gestión refuerzan la idea de la calidad como instrumento clave en la busca de la supervivencia en un mercado competitivo. El principio básico de la filosofía de la gestión de la calidad debe estar orientado a la satisfacción del usuario, en la busca de motivación, en el compromiso de los profesionales y de todos los colaboradores y en la integración e interrelación en los procesos de trabajo. Un enfoque dinámico, contínuo y participativo, donde debe estar implícita la responsabilidad personal de todos los miembros de la organización en el desarrollo de nuevas formas de información y comunicación, orientado a la implementación de la efectividad, eficiencia y lucro en los procesos que aportan valor agregado y oculto a la organización y a los usuarios. ${ }^{(2)}$

Uno de los conceptos relacionados con la calidad es el de evaluación, destacando la importancia de la acreditación hospitalaria, una herramienta que contiene criterios que colaboran y estimulan la mejora de la calidad, siendo un proceso en el cual una entidad, separada e independiente de la institución de salud, evalúa la institución de salud para 
determinar si ella cumple una serie de estándares creados para perfeccionar la seguridad y calidad del cuidado, propiciando la creación de una cultura de seguridad y calidad en el interior de una institución que se empeña en perfeccionar continuamente los métodos de prestación de cuidados al paciente y los resultados obtenidos. ${ }^{(3)}$ Es un proceso que estimula a las instituciones implicadas para un comportamiento saludable que procure la mejora contínua de la calidad de la asistencia prestada. ${ }^{(4)}$

Ante este contexto y dada la importancia de la temática, se resolvió investigar el asunto, con el propósito de ayudar al proyecto de investigación "Estudio del proceso de acreditación en una institución de salud de la red pública del Estado de Rio de Janeiro" que está siendo desarrollado junto al Programa de Mestrado Académico en Ciencias del Cuidado en Salud de la Escuela de Enfermería Aurora de Afonso Costa de la Universidad Federal Fluminense (UFF) y por entender que la Enfermería es una categoría profesional que se preocupa de la calidad, estando siempre dispuesta a aprender, mejorar e implementar un proceso de calidad. (4)

Este estudio tiene por objetivo verificar cómo la temática acreditación y/o evaluación de los servicios de salud está siendo abordada en la literatura.

\section{METODOLOGÍA}

El presente estudio es una revisión integrativa de la literatura, realizada a través de la Biblioteca Virtual de Saúde (BVS). Este método de investigación permite la síntesis de múltiples estudios publicados posibilitando conclusiones generales sobre un área particular. (5)

Se realizó la investigación en las bases de datos electrónicas: LILACS, IBECS, BEDENF y MEDLINE, utilizándose los descriptores: Acreditación, Calidad de la asistencia a la salud, acceso y evaluación.

Los criterios de inclusión utilizados en este estudio fueron: artículos de periódicos disponibles íntegramente online, en portugués, español o inglés, con un recorte temporal entre los años de 2005 a 2010. Enriqueció la presente revisión, una disertación de mestrado, la cual constaba en la base de datos LILACS, por eso no estaba disponible online, siendo necesario su rescate en la biblioteca de la Universidad Estadual do Rio de Janeiro-Brasil, debido a su relevancia para el estudio.

La cuestión que dirigió la busca de los artículos fue: ¿Qué dice la literatura sobre acreditación y/o evaluación de los servicios de salud?

\section{RESULTADOS Y DISCUSIÓN}

El resultado de la busca en las bases de datos de la BVS se presentó de la siguiente forma: Con todos los descriptores simultáneamente no se encontraron resultados; utilizándose cualquier descriptor, encontramos: Base de datos LILACS 317, IBECS 148 y MEDLINE 10081.

Tras este levantamiento, utilizamos el filtrado con los criterios de inclusión propuestos para la investigación contraponiéndolos con la pregunta guía. Se seleccionaron entonces 18 artículos íntegramente online y una disertación de mestrado que ayudaron al estudio. Hay que destacar que un artículo de la base de datos MEDLINE se repetía en LILACS. 
De los 19 estudios analizados, 02 se publicaron en 2010, 03 en 2009, 06 en 2008, 02 en 2007, 04 en 2006 y 02 en 2005.

En cuanto a las fuentes de datos, se constató que las fuentes de datos Brasileñas presentaron el mayor número de publicaciones con 08, siendo 03 en el Estado de São Paulo, 02 en Brasília, 02 en Rio de Janeiro y 01 en Minas Gerais. Los Estados Unidos tuvieron 05; Bolivia 03; Chile, Inglaterra e Italia tuvieron 01 cada uno.

En relación a la calificación de los autores de los estudios, se identificó que 41,5\% de los profesionales son médicos, $24,6 \%$ son enfermeros, $1,5 \%$ es administrador y $34,3 \%$ no fueron identificados.

En relación al diseño de la investigación, los estudios evidenciados más frecuentes fueron: enfoque cuantitativo en 07 estudios; enfoque cualitativo en 03 estudios; Revisión de literatura en 04 estudios; 03 Instrumentos normativos; enfoque cualitativo-cuantitivo en 01 estudio y se encontró una carta al editor.

Las investigaciones se desarrollaron en diversos escenarios, siendo 18 en establecimientos de salud relacionados con el nivel secundario y terciario y 01 en el nivel primario.

Para analizar los datos encontrados, utilizamos la lectura interpretativa y análisis temático. Emergieron de este análisis las siguientes categorías:

1. Criterios de resultado y proceso de evaluación

2. Beneficios para la asistencia

3. Cambio cultural.

\section{Criterios de resultado y proceso de evaluación}

En esta categoría se incluyen 08 artículos y 01 disertación de mestrado que tratan de los criterios de resultado y proceso de evaluación en la acreditación hospitalaria, conforme tabla I. 
Tabla I. Distribución de las bibliografías potenciales de la categoría temática "Criterios de resultado y proceso de evaluación"

\begin{tabular}{|c|c|c|c|}
\hline AUTORES & ANO & $\begin{array}{l}\text { BASE DE DADOS/FONTE DE } \\
\text { DADOS }\end{array}$ & TÍTULO \\
\hline $\begin{array}{l}\text { Shaw; Kutryba; } \\
\text { Braithwaite; Bedlicki; } \\
\text { Andrzejwarunek }^{(6)}\end{array}$ & 2010 & $\begin{array}{l}\text { MEDLINE/ Intemational Joumal for } \\
\text { Quality in Health Care }\end{array}$ & $\begin{array}{l}\text { Sustainable healthcare } \\
\text { accreditation: } \\
\text { messages from Europe in } \\
2009\end{array}$ \\
\hline $\begin{array}{l}\text { Chung-I; Cathy; } \\
\text { Yang }(7)\end{array}$ & 2009 & $\begin{array}{l}\text { MEDLINE/ BMC Health Services } \\
\text { Research }\end{array}$ & $\begin{array}{l}\text { Developing } 21 \text { st century } \\
\text { accreditation standards } \\
\text { for teaching hospitals: the } \\
\text { Taiwan experience }\end{array}$ \\
\hline $\begin{array}{l}\text { Bolívia. Ministério da } \\
\text { saúde e do desporto }\end{array}$ & 2008 & $\begin{array}{l}\text { LILACS/ La Paz, Bolívia. Ministério da } \\
\text { saúde e do desporto }\end{array}$ & $\begin{array}{l}\text { Manual de evaluación y } \\
\text { acreditación de } \\
\text { establecimientos de salud } \\
\text { tercer nivel de atención }\end{array}$ \\
\hline $\begin{array}{l}\text { Bolívia. Ministério da } \\
\text { saúde e do desporto }\end{array}$ & 2008 & $\begin{array}{l}\text { LILACS/ La Paz, Bolívia. Ministério da } \\
\text { saúde e do desporto }\end{array}$ & $\begin{array}{l}\text { Manual de evaluación y } \\
\text { acreditación de } \\
\text { establecimientos de salud } \\
\text { segundo nivel }\end{array}$ \\
\hline $\begin{array}{l}\text { Bolivia. Ministério da } \\
\text { saúde e do desporto }\end{array}$ & 2008 & $\begin{array}{l}\text { LILACS/ La Paz, Bolivia. Ministério da } \\
\text { saúde e do desporto }\end{array}$ & $\begin{array}{l}\text { Manual de evaluación y } \\
\text { acreditación de } \\
\text { establecimientos de salud } \\
\text { primer nivel de atención }\end{array}$ \\
\hline Fortes $(11)$ & 2007 & $\begin{array}{l}\text { LILACS/ Universidade do Estado do } \\
\text { Rio de Janeiro [dissertação mestrado] }\end{array}$ & $\begin{array}{l}\text { A acreditação no } \\
\text { contexto dos sistemas de } \\
\text { saúde: as propostas de } \\
\text { política e suas diversas } \\
\text { acreditações }\end{array}$ \\
\hline Feldman; Cunha ${ }^{(12)}$ & 2006 & $\begin{array}{l}\text { MEDLINE/ Revista Latino Americana } \\
\text { em Enfermagem }\end{array}$ & $\begin{array}{l}\text { Identificação dos critérios } \\
\text { de avaliação de } \\
\text { resultados do Serviço de } \\
\text { enfermagem nos } \\
\text { programas de acreditação } \\
\text { hospitalar }\end{array}$ \\
\hline Cunha; Feldman ${ }^{(13)}$ & 2005 & $\begin{array}{l}\text { MEDLINE/ Revista Brasileira de } \\
\text { Enfermagem }\end{array}$ & $\begin{array}{l}\text { Avaliação dos Serviços de } \\
\text { enfermagem: Identificação } \\
\text { dos critérios de processo } \\
\text { dos programas de } \\
\text { acreditação hospitalar }\end{array}$ \\
\hline $\begin{array}{l}\text { Moreno Júnior; } \\
\text { Zucchi }(14)\end{array}$ & 2005 & $\begin{array}{l}\text { LILACS/ Revista Administração } \\
\text { Pública }\end{array}$ & $\begin{array}{l}\text { Avaliação de qualidade } \\
\text { em serviços de saúde: } \\
\text { acreditação, certificação e } \\
\text { programas de melhoria da } \\
\text { qualidade em hospitais } \\
\text { públicos e privados do } \\
\text { município de São Paulo }\end{array}$ \\
\hline
\end{tabular}

Fonte: BVS, 2010

El estudio llevado a cabo en Europa ${ }^{(6)}$ describe el desarrollo de las organizaciones nacionales de acreditación en relación a los incentivos, financiación y posición en el mercado en 2009, identificando las tendencias utilizando datos de investigaciónes anteriores. Se detectó que los principales desafíos para una acreditación sustentable parecen estar en el tamaño del mercado, en la coherencia del apoyo de la política, en la financiación de programas e incentivos financieros para la participación. 
El estudio realizado en Taiwan ${ }^{(7)}$ establece estándares de enseñanza de la acreditación hospitalaria con la esperanza de que los hospitales de enseñanza del país pudiesen superar las expectativas de la sociedad taiwandesa y garantizar la calidad de la enseñanza. Esperan que las nuevas normas refuercen la enseñanza y la investigación, y mejoren la calidad de los servicios hospitalarios, al mismo tiempo.

Los manuales de evaluación y acreditación de los establecimientos de salud elaborados en Bolivia, Ministerio de la Salud y del Deporte ${ }^{(8,9,10)}$ son documentos técnicos normativos que proponen establecer um sistema de evaluación y acreditación que contribuya a la mejora continua y sostenida de la calidad de atención de los establecimientos del Sistema Nacional de Salud de Bolivia.

La disertación ${ }^{(11)}$ analizó las relaciones de la acreditación dentro de los sistemas de salud y verificó la posición de la Organización Mundial de la Salud (OMS) en relación al tema acreditación. A partir del análisis documental quedaron claras las dimensiones de la acreditación como producto de mercado y mecanismo que propicia la visibilidad de las innovaciones promovidas en los sistemas de salud en pro del compromiso con la calidad. Se desarrollaron dos maneras de adhesión a la acreditación, los imperativos de mercado y las formas de condicionalidad u obligatoriedad impuestas por el gobierno o por uno de sus órganos reguladores, a través de sus políticas. Se percibió que el cálculo que permitiría anticipar la relación de la acreditación con los sistemas de salud no aparece desarrollado plenamente en la reflexión de la OMS. Del análisis comparativo de los sistemas de salud francés, inglés y español teniendo la acreditación como base, se verificó que no hay una sóla acreditación. En Francia, por ejemplo, no parece el carácter voluntario, inherente a la naturaleza de la acreditación. En este país, la acreditación es obligatoria.

Dos estudios realizados en Brasil ${ }^{(12,13)}$ identificaron los criterios de evaluación de resultado y de proceso aplicados al servicio de enfermería adoptados en los programas de acreditación y los clasificó en las atribuciones: administrativa, asistencial, enseñanza/investigación. Los autores verificaron que el servicio de enfermería es evaluado con énfasis en las atribuciones administrativas, indicando que los criterios relativos a las atribuciones asistenciales y enseñanza/investigación todavía necesitan ser reevaluados en los aspectos cuantitativos y cualitativos para obtener una medida más precisa de la prestación del cuidado. En la etapa de selección de los criterios de evaluación del servicio de enfermería, encontraron dificultad una vez que la literatura no dispone de instrumentos de esta clasificación. Se basaron en la propia experiencia de evaluación de la investigadora, así como en las reflexiones y asesoramientos de la orientadora, para definir la posición de los criterios en las atribuciones, de la forma más adecuada. Se sabe que los criterios de evaluación, en general, tienen un movimiento propio, por tanto debe ser flexible y capaz de adecuarse a las necesidades locales y regionales en cada establecimiento de salud, suficientemente específicos para responder a los estándares preestablecidos. Constantemente son necesarias reflexiones, sugerencias y críticas para atender las propuestas evaluadoras.

Otro estudio brasileño ${ }^{(14)}$ identificó los métodos de evaluación de la calidad utilizados en 12 establecimentos hospitalarios públicos y privados del municipio de São Paulo. Señaló la existencia de programas de mejora de la calidad, relacionando los factores citados con los problemas para su implantación en esos mismos hospitales. Evidenció la preferencia por el uso de la acreditación hospitalaria como metodología estructurada de evaluación y la cultura organizacional como el principal problema relacionado con la implantación de la calidad. Concluye que la calidad en el sector de salud ha presentado una expansión creciente de acciones que miran a la garantía y a la mejora continua y que alimentan, principalmente, las 
exigencias de la competitividad de mercado y de los usuarios de los servicios. Es, por tanto, primordial la implementación de programas permanentes de calidad y la adecuada gestión de los recursos del sistema para la mejora de los resultados de las organizaciones de salud.

Discutiendo esta categoría se destaca que la implementación de la acreditación como herramienta para evaluación de la gestión de la calidad apunta a la mejora de la calidad de los servicios hospitalarios prestados, propicia visibilidad de las innovaciones promovidas en los sistemas de salud en pro del compromiso con la calidad. Los estudios evidenciaron la preferencia por el uso de la acreditación hospitalaria como metodología estructurada de evaluación. En lo que se refiere al Servicio de Enfermería en este proceso, los estudios mostraron que el servicio es evaluado con énfasis en las atribuciones administrativas, mas precisa ser mejorado en los criterios relativos a las atribuciones asistenciales y enseñanza/invesigación, necesitando de ajustes, reflexión y aumento de criterios para una evaluación más precisa de la prestación del cuidado.

\section{Benefícios para la asistencia}

En esta categoría se incluyen 07 artículos que tratan sobre los beneficios para la asistencia, conforme tabla II. 
Tabla II. Distribución de las bibliografías potenciales de la categoría temática "beneficios para la asistencia"

\begin{tabular}{|c|c|c|c|}
\hline AUTORES & ANO & $\begin{array}{l}\text { BASE DE DADOS/FONTE DE } \\
\text { DADOS }\end{array}$ & TÍTULO \\
\hline Auras, Geraedts $(15)$ & 2010 & $\begin{array}{l}\text { MEDLINE/Intemational Joumal for } \\
\text { Quality in Health Care }\end{array}$ & $\begin{array}{l}\text { Patient experience data } \\
\text { in practice } \\
\text { Accreditation - an } \\
\text { intemational } \\
\text { comparison }\end{array}$ \\
\hline $\begin{array}{l}\text { Greenfield; } \\
\text { Pawsey; Naylor; } \\
\text { Braithwaite }^{(16)}\end{array}$ & 2009 & $\begin{array}{l}\text { MEDLINE/Intemational Joumal of } \\
\text { Health Care Quality Assurance }\end{array}$ & $\begin{array}{l}\text { Are accreditation } \\
\text { surveys reliable? }\end{array}$ \\
\hline $\begin{array}{l}\text { Tehewy; Salem; } \\
\text { Habil; Okda }\end{array}$ & 2009 & $\begin{array}{l}\text { MEDLINE/Intemational Joumal for } \\
\text { Quality in Health Care }\end{array}$ & $\begin{array}{l}\text { Evaluation of } \\
\text { accreditation program } \\
\text { in non-governmental } \\
\text { organizations' health } \\
\text { units in Egypt: short- } \\
\text { term outcomes }\end{array}$ \\
\hline Aretxabala $a^{(18)}$ & 2008 & LILACS/ Revista Médica do Chile & $\begin{array}{l}\text { Oportunidades y } \\
\text { desafios en el sistema } \\
\text { de salud: [carta al } \\
\text { editor] }\end{array}$ \\
\hline $\begin{array}{l}\text { Saghatchian, } \\
\text { Hummel, Otter, } \\
\text { Valeriola, Harten, } \\
\text { Paradiso, Koot, } \\
\text { Tursz } \\
\text { (19) }\end{array}$ & 2008 & MEDLINE/ Tumori & $\begin{array}{l}\text { Towards quality } \\
\text { comprehensiveness } \\
\text { and excellence } \\
\text { comprehensiveness } \\
\text { and excellence }\end{array}$ \\
\hline $\begin{array}{l}\text { El-Jardali, Jamal, } \\
\text { Dimassi, Ammar, } \\
\text { Tchaghchaghia }{ }^{(20)}\end{array}$ & 2008 & $\begin{array}{l}\text { MEDLINE/Intemational Joumal for } \\
\text { Quality in Health Care }\end{array}$ & $\begin{array}{l}\text { The impact of hospital } \\
\text { accreditation on } \\
\text { quality of care: } \\
\text { perception of } \\
\text { Lebanese nurses }\end{array}$ \\
\hline $\begin{array}{l}\text { Matos, Morais, } \\
\text { Neumann, Santos, } \\
\text { Silva, Alves }\end{array}$ & 2006 & $\begin{array}{c}\text { LILACS/ REME - Revista Mineira de } \\
\text { Enfermagem }\end{array}$ & $\begin{array}{l}\text { Um olhar sobre as } \\
\text { ações do enfermeiro } \\
\text { no Processo de } \\
\text { Acreditação hospitalar }\end{array}$ \\
\hline Lima; Erdmann ${ }^{(22)}$ & 2006 & LILACS/Acta Paulista de Enfermagem & $\begin{array}{l}\text { A enfermagem no } \\
\text { processo da } \\
\text { acreditação hospitalar } \\
\text { em um serviço de } \\
\text { urgência e emergência }\end{array}$ \\
\hline
\end{tabular}

Fonte: BVS, 2010

Un estudio realizado en Europa ${ }^{(15)}$ describe el método, contenido y alcance, así como diferencias, semejanzas y características únicas en la integración de datos de la experiencia del paciente en la prática internacional de los programas de acreditación. Detectó que la colecta de datos sobre la experiencia del paciente es de gran importancia para una evaluación global de la calidad de los cuidados médicos. Un programa de acreditación, que incluye una investigación estructurada para el paciente, puede considerarse como un alto estándar internacional. Hasta ahora, existen resultados de investigaciones suficientes sobre cómo y en qué medida los datos de la experiencia del paciente deben incluirse en la decisión de acreditación. 
Un estudio australiano ${ }^{(16)}$ testó si los procesos de levantamiento de la acreditación de cuidados de salud son confiables. Identificó 6 factores interrelacionados que forman la confiabilidad - la influencia que ellos ejercen simultáneamente promueve y desafía la consistencia de la investigación. Detectó que 'son necesarias otras investigaciones para comprender los meandros de interrelaciones entre estos factores, cuantificar en resultados mensurables a los evaluadores y la confiabilidad en los aspectos de las reglas del grupo de investigación. A través de un análisis de estos factores, argumentó que la confiabilidad es construida en el proceso de acreditación. Por eso no es de extrañar que el esfuerzo de la creación del juicio de la confiabilidad de las condiciones de acreditación sea desafiante y complejo.

Un estudio realizado en Egipto ${ }^{(17)}$ objetivó determinar el efecto de la acreditación en las unidades de salud de las Organizaciones no gubernamentales (ONGs) en lo que se refiere a satisfacción del paciente y satisfacción del prestador, y de la producción de la acreditación en el cumplimiento de algunas normas de acreditación. Detectó que los pacientes atendidos en las entidades acreditadas ONGs expresaron resultados de satisfacción significativamente mayores de los que van a las unidades no-credenciadas. Este estándar se observó en todas las áreas del servicio de salud. No mostró ninguna diferencia significativa en el grado medio de satisfacción entre la intervención y grupos control en relación al ambiente social, administrativo y al modelo de salud de familia. Globalmente, el grupo de proveedores acreditados no mostró un grado de satisfacción significativamente mayor comparado con las unidades de salud no acreditadas. Así la acreditación, durante los primeros meses de su aplicación puede mejorar la satisfacción de los prestadores de servicios no a través del ambiente social o administrativo, sino porque adapte el sistema de salud o a través de los aspectos no identificados en la investigación. Mostró que elementos de estructura representaron mayor porcentaje en unidades acreditadas significando que son mejores de alcanzar y mantener en comparación con medidas de tratamiento del paciente. La principal diferencia entre unidades acreditadas y no acreditadas fue el mantenimiento de los registros por lo que la acreditación puede ser el principal factor subyacente al mejor desempeño de unidades acreditadas en el acompañamiento de pacientes crónicos. Se detectó que las unidades de salud acreditadas siguen cumpliendo las normas de acreditación en el primer año tras la obtención de la acreditación. Había una limitación de intervención pre-medidas no disponible (pre-acreditación) recomendándose que se realicen futuras investigaciones con control preconcebido y posconcebido para evaluar el efecto de la acreditación en los servicios de salud.

La carta al editor ${ }^{(18)}$ mostró cómo el cambio de la Ley en el Sistema Nacional de Salud de Chile afectará directamente la actuación médica y hará que la comunidad médica de Chile conozca la norma de acreditación y se involucre con este tema, por ser algo que les atañe directamente. Hacer de esta Ley una oportunidad y un desafío para la mejora de la atención a los pacientes.

Un estudio realizado en Europa ${ }^{(19)}$ abordó aspectos relevantes del proyecto de Acreditación de la Organización Europea de Institutos de Cáncer (OECI) que tiene como objetivos: desarrollar un sistema de acreditación global de cuidados en oncología, contando con la prevención, asistencia, investigación, educación y networking; definir un banco de datos actualizado de los centros de cáncer en Europa, con informaciones completas sobre sus recursos y actividades (en la asistencia, investigación, educación y gestión) y desarrollar una herramienta global de etiquetado específica para centros integrales de cáncer en Europa, que designe los varios tipos de estructuras de cáncer, y los centros detallados de cáncer de referencia y excelencia. El estudio destaca que entre los beneficios potenciales del proyecto, los más inmediatos son: mejor atención a las personas; reforzar la confianza de la 
comunidad en la calidad de los cuidados continuados en el hospital; salud y educación profesional; estimulación de los esfuerzos de mejora de la calidad, si las recomendaciones de acreditación son aplicadas tras el proceso de acreditación; evaluación objetiva de la calidad de los cuidados del hospital; potencial para mejorar la cobertura del seguro de responsabilidad; evaluación comparativa de las estructuras de cuidados; fortalecimiento de una visión más global y coherente con una base de evidencia clara; información al público con una mayor armonización y equidad para los pacientes. Concluye que la evaluación de estructuras de cáncer puede servir de base para la evaluación y acreditación de diferentes especialidades relacionadas con el tratamiento del cáncer, como cirugía, radioterapia, técnicas de imagen, estructuras de rehabilitación, la medición de resultados y los registros de salud. El proyecto de acreditación OECI podría ser la fundación de una nueva alianza entre los centros de cáncer y sus socios en el progreso continuo y busca de la excelencia de la investigación y tratamiento en oncología.

Un estudio libanés ${ }^{(20)}$ evaluó el potencial impacto de la acreditación en la calidad del cuidado a través del prisma de profesionales de salud, específicamente enfermeros. Investigó también la percepción de los factores que contribuyen a explicar los cambios en la calidad del tratamiento. Esta evaluación tuvo en cuenta el tamaño de los hospitales, ya que pueden tener impacto en la implementación de la mejora de la calidad. Las enfermeras percibieron una mejora de los resultados de calidad en los hospitales como un resultado de la acreditación. Percibieron el trabajo en equipo y la mejora de la productividad en los hospitales como un resultado de la acreditación. Recompensas y reconocimiento obtuvieron la puntuación más baja de acuerdo. El modelo indicó que los predictores de mejor calidad en los resultados fueron liderazgo, compromiso, soporte, uso de datos y gestión de la calidad, compromiso personal y el tamaño del hospital. Para los hospitales de grande y pequeño tamaño, el impacto de la escala de medida de gestión de la calidad no mostró significado estadístico. La participación de los funcionarios en la Acreditación tuvo mayor impacto en hospitales de pequeño porte. Para hospitales de grande y de medio tamaño de personal, la participación no presentó significado, con resultados de calidad. Esta observación puede indicar que el personal del hospital de pequeño tamaño estaba más involucrado en la acreditación, y tal participación ayudó a los hospitales a mejorar sus resultados de calidad. La evidencia muestra que las organizaciones de menor dimensión tienen frecuentemente una cultura más homogenea y su equipo probablemente comparte los mismos valores. Hospitales de gran tamaño tienden a ser organizados más jerárquica y burocráticamente, lo que dificulta la concreción del trabajo de calidad. Concluye que la acreditación hospitalaria, según las enfermeras del Líbano, es una buena herramienta para mejorar la calidad del tratamiento. Para hacer de la acreditación un instrumento eficaz de reglamentación, hay una necesidad de evaluar la calidad con base en indicadores de resultados del paciente. Esto puede hacerse a través del refuerzo del programa de acreditación en curso más orientado para resultados. Los resultados del estudio también proporcionarán valiosas lecciones para otros países de la región que están preparando o están ejecutando la acreditación.

Un estudio realizado en Brasil ${ }^{(21)}$ describió acciones del enfermero en el proceso de acreditación hospitalaria. Se identificaron 1889 referencias, de las cuales 15 estaban directamente relacionadas con el papel del enfermero en la acreditación. Con relación a la acreditación, se observó que la mayoría de las referencias encontradas se relacionaba con sectores específicos del hospital, como, Centro Quirúrgico, Laboratorio de Patología, Centro de Tratamiento Intensivo, Farmacia, etc. Solo una minoría trataba la acreditación del hospital como un todo. Concluye que el liderazgo, la comunicación, la habilidad técnico-científica, la organización del trabajo y los programas de educación continuada son estrategias que posibilitan al enfermero implementar los cambios requeridos a las nuevas situaciones y al trabajo interdisciplinar. De esta forma este profesional proporciona mejora en la calidad de la 
asistencia contribuyendo efectivamente al alcance de los objetivos institucionales, al éxito del proceso de acreditación.

Otro estudio brasileño ${ }^{(22)}$ objetivó construir acciones de enfermería para reestructurar las Urgencias de un hospital universitario de acuerdo con los estándares de la Acreditación Hospitalaria en urgencia y emergencia según el Ministerio de Salud. Fue posible verificar que el cuidado de enfermería es realizado por la acción/negociación/deliberación de sus profesionales frente a las necesidades levantadas. De este modo, los ítems del nivel 1 de la Acreditación Hospitalaria fueron todos revisados uno a uno, siendo los listados renegociados, deliberados y otros implementados por el grupo, si no en el momento, con cronograma prefijado. Los ítems elegido para reestructuración fueron cuatro: Equipo capacitado para atención en urgencia y emergencia, registro de las acciones asistenciales a los pacientes en observación, equipamentos, medicamentos y materiales compatibles con la estructura del servicio y acompañantes o responsables informados sobre el estado general del paciente asistido. El estudio posibilitó reflexiones en busca de la calidad en el cuidado de enfermería, instrumentalizando el equipo en busca de la Acreditación Hospitalaria, con vistas a la mejora de la calidad de la asistencia. Acreditamos que los enfermeros pueden desarrollar programas innovadores en las organizaciones, centrados en nuevas concepciones de estructura y propiedades de sus servicios para obtener mejores prácticas en salud y mejor calidad del cuidado, principalmente, en hospitales universitarios, espacio de formación de los futuros profesionales que trabajarán en la sociedad.

Discutiendo esta categoría se destaca que la confiabilidad de la acreditación es construida en el transcurso del proceso de implementación y validación, el tema de la confiabilidad es desafiante y complejo, siendo necesarios más estudios para la comprensión de los factores interrelacionados que forman la confiabilidad. Los estudios señalaron que los pacientes atendidos en las instituciones acreditadas expresaron resultados de satisfacción significativamente mayores que los que van a instituciones no acreditadas, por ello se recomienda la realización de futuras investigaciones con control pre y pos acreditación realizadas para evaluar el efecto de la acreditación en los servicios de salud. Se detectaron beneficios potenciales del proyecto de acreditación: mejor atención a las personas, refuerza la confianza de la comunidad en la calidad del cuidado. En lo que se refiere a enfermería, los estudios indicaron que las enfermeras percibieron una mejora en los resultados de la calidad con la acreditación, siendo necesario evaluar la calidad basándonos en evidencias de resultado del paciente. Posibilitó reflexiones en busca de la Acreditación Hospitalaria con vistas a la mejora de la calidad de la asistencia.

\section{Cambio Cultural}

En esta categoría se incluyen 02 artículos que tratan sobre cambio cultural, conforme tabla III. 
Tabla III. Distribución de las bibliografías potenciales de la categoría temática "cambio cultural"

\begin{tabular}{l|c|c|l}
\hline \multicolumn{1}{c|}{ AUTORES } & ANO & $\begin{array}{c}\text { BASE DE DADOS/FONTE DE } \\
\text { DADOS }\end{array}$ & \multicolumn{1}{c}{ TÍTULO } \\
\hline Novaes (23) & 2007 & $\begin{array}{c}\text { LILACS/Revista Administração em } \\
\text { Saúde }\end{array}$ & $\begin{array}{l}\text { O processo de } \\
\text { acreditação dos } \\
\text { serviços de saúde }\end{array}$ \\
\hline $\begin{array}{l}\text { D'Innocenzo, } \\
\text { Adami, Cunha (24) }\end{array}$ & 2006 & $\begin{array}{c}\text { LILACS/Revista Brasileira de } \\
\text { Enfermagem }\end{array}$ & $\begin{array}{l}\text { O movimento pela } \\
\text { qualidade nos serviços } \\
\text { de saúde e } \\
\text { enfermagem }\end{array}$ \\
\hline
\end{tabular}

Fonte: BVS, 2010

Un estudio brasileño ${ }^{(23)}$ relató características de los conceptos de Acreditación de hospitales y sus aspectos evolutivos. Detectó que inicialmente, los estándares referenciales para los hospitales son en general establecidos con base en 'estructura' de las instituciones; posteriormente son evaluados aspectos de procedimientos o 'procesos' y, finalmente, en la evaluación de los 'resultados' institucionales y de sus impactos en la comunidad.

En la actualidad, principalmente en los EUA, el enfásis de los programas de evaluación de la calidad está en los aspectos de seguridad del paciente, con el objetivo de evitar incompetencias médicas o graves errores en la asistencia hospitalaria. De la misma forma que en los EUA solamente los hospitales que presentan indicadores de buena calidad reciben recursos del gobierno federal; esperamos que en un futuro no distante los recursos de nuestro SUS sean transferidos solamente a los hospitales certificados. Concluye que la acreditación evoluciona continuamente y agrega nuevas iniciativas para aumentar la calidad de la atención progresivamente. Debe aceptarse que ella, por sí sola, no evita la ocurrencia de errores profesionales, y que la probabilidad de fallos es alta. Es preciso reconocerlos y detectar sus orígenes. Para disminuir estos eventos es necesario, muchas veces, cambiar la cultura institucional, rediseñar los sistemas, reevaluar los procesos directamente involucrados en los fallos y evitar el cansancio de los profesionales. Finalmente, nunca se podrá implantar cualquier programa de acreditación si el cuerpo clínico no está cualificado y, probablemente, los aspectos de formación, certificación y recertificación de la profesión médica en América Latina serán, tal vez, el mayor desafío del sector salud, en este nuevo siglo. Repitamos lo que Sir Liam Donaldson, ministro inglés, afirmó: "Errar es humano. Ocultar los errores es imperdonable. No aprender de ellos no tiene perdón”. ¿Nuestros servicios de salud estarán preparados para promover una nueva cultura y romper con la cultura del silencio? ${ }^{(23: 139)}$

Otro estudio brasileño ${ }^{(24)}$ describe la trayectoria del movimiento mundial por la calidad y su inserción en los servicios de salud, puntuando el referencial teórico de Donabedian y, abordando la Sistematización de la Asistencia de Enfermería como base para la calidad de esa asistencia prestada al usuario. Destacó que el movimiento por la calidad en los servicios de salud y Enfermería es una necesidad incorporada a la gestión de esas áreas a fin de asegurar la asistencia libre de riesgos al usuario. Concluye que en Enfermería, donde el cuidar es la esencia de la profesión, la concentración de esfuerzos dirigidos a los objetivos propuestos deberá conducir a una mejora continua de la atención. Esto implica la concienciación de todo el equipo en cuanto a su importancia y al valor de sus acciones. Compromiso, cooperación, dedicación y mejora continua de enfermería deberán llevar a los resultados deseados para el paciente y para los profesionales. 
Discutiendo esta categoría se destaca que la acreditación evoluciona y agrega nuevas iniciativas para aumentar la calidad de la atención, siendo necesario un cambio en la cultura institucional, pues no evita la ocurrencia de errores profesionales, siendo preciso reconocerlos y detectar sus orígenes. En lo que se refiere a la Enfermería, el movimiento por la calidad es una necesidad incorporada a la gestión a fin de asegurar la asistencia libre de riesgos al usuario, lo que implica la concienciación de todo el equipo.

\section{CONCLUSIÓN}

Se concluye que la acreditación de los servicios de salud es una herramienta que está siendo utilizada mundialmente, en algunos países con adhesión voluntaria y en otros obligatoria por el gobierno. La acreditación evoluciona sus procesos continuamente para alcanzar con excelencia sus objetivos. A pesar de no evitar la ocurrencia de errores profesionales, ha sido una oportunidad de las instituciones de salud para mejorar la calidad del centro de contacto, atención y cuidado al paciente. Se identifica la necesidad de un cambio cultural en las instituciones de salud, cualificación de los profesionales, liderazgo, compromiso, entre otros factores, para el cumplimiento y el éxito del proceso. En lo que se refiere al Servicio de Enfermería, el estudio permitió identificar su importante participación en el proceso, anotando la necesidad de ajustes para una evaluación más efectiva de la prestación del cuidado y concienciación de todo el equipo.

\section{REFERENCIAS}

1. Leitão RER, Kurcgant, P. Qualidade na prática gerencial da Enfermagem: as duas faces da mesma moeda. Niterói: Intertexto; 2004.

2. Feldman LB. Gestão de Risco e Segurança Hospitalar. São Paulo: Martinari; 2008.

3. JCl. Padrões de acreditação da Joint Commision International para Hospitais. $3^{\mathrm{a}}$ ed. Rio de Janeiro; 2008.

4. Soares de Lima SB, Erdman AL, Prochnow AG, Leite JL, Moreira MCh. Percepção dos enfermeiros do serviço de urgência e emergência em relação à acreditação hospitalar. Enfermería Global número 11. noviembre 2007. Revista electrónica semestral de enfermería. 5. Mendes KDS, Silveira RCCP, Galvão CM. Revisão integrativa: método de pesquisa para a incorporação de evidências na saúde e na Enfermagem. Texto Contexto Enferm. 2008 Out-Dez; 17(4): 758-764.

6. Shaw CD, Kutryba B, Braithwaite J, Bedlicki M, Warunek A. Sustainable healthcare accreditation: messages from Europe in 2009. International Journal for Quality in Health Care. 2010; 22 (5): 341-350.

7. Chung-I H, Cathy W, Yang Che-M. Developing 21 st century accreditation standards for teaching hospitals: the Taiwan experience. BMC Health Serv Res. 2009; 9:232.

8. Bolívia. Ministério da saúde e do desporto. Manual de evaluación y acreditación de establecimientos de salud tercer nivel de atención. La Paz, Bolívia. Ministério da saúde e do desporto. 2009.

9. Bolívia. Ministério da saúde e do desporto. Manual de evaluación y acreditación de establecimientos de salud segundo nivel. La Paz, Bolívia. Ministério da saúde e do desporto. 2009.

10. Bolívia. Ministério da saúde e do desporto. Manual de evaluación y acreditación de establecimientos de salud primer nivel de atención . La Paz, Bolívia. Ministério da saúde e do desporto 2009.

11. Fortes MTR. A acreditação no contexto dos sistemas de saúde: as propostas de política e suas diversas acreditações [dissertação mestrado]. Rio de Janeiro: Universidade do Estado do Rio de Janeiro; 2007. 
12. Feldman LB, Cunha ICKO. Identificação dos critérios de avaliação de resultados do Serviço de enfermagem nos programas de acreditação hospitalar. Rev Latino-am Enfermagem. 2006 Jul-Ago; 14(4): 540-545.

13. Cunha ICKO, Feldman LB. Avaliação dos serviços de enfermagem: identificação dos critérios de processo dos programas de acreditação hospitalar. Rev Bras Enferm. 2005 JanFev; 58(1): 65-69.

14. Moreno Junior JMP, Zucchi P. Avaliação de qualidade em serviços de saúde: acreditação, certificação e programas de melhoria da qualidade em hospitais públicos e privados do município de São Paulo. RAP. 2005 Jan-Fev; 39(1): 7-16.

15. Auras S, Geraedts M. Patient experience data in practice accreditation-an international comparison. International Journal for Quality in Health Care. 2010 Fev; 22(2):132-139.

16. Greenfield $D$ et al. Are accreditation surveys reliable? International Journal of Health Care Quality Assurance. 2009; 22(2): 105-116.

17. Tehewy Mal, Salem B, Habil I, Sayed EO. Evaluation of accreditation program in nongovernmental organizations' health units in Egypt: short-term outcomes. International Journal for Quality in Health Care. 2009; 21(3): 183-189.

18. Aretxabala X. Oportunidades y desafíos en el sistema de salud: [carta al editor]. Rev Méd Chile. 2008; 136(12):1604.

19. Saghatchian $M$ et al. Towards quality comprehensiveness and excellence comprehensiveness and excellence. Tumori. 2008; 94:164-171.

20. El-Jardali $\mathrm{F}$ et al. The impact of hospital accreditation on quality of care: perception of Lebanese nurses. International Journal for Quality in Health Care. 2008; 20(5): 363-371.

21. Matos SS et al. Um olhar sobre as ações do enfermeiro no Processo de Acreditação hospitalar. REME - Rev Min Enf. 2006 Out-Dez; 10(4): 325-337.

22. Lima SBS, Erdmann AL. A enfermagem no processo da acreditação hospitalar em um serviço de urgência e emergência. Acta Paul Enf. 2006 Jul-Ago; 19(3): 271-278.

23. Novaes HM. O processo de acreditação dos serviços de saúde. Rev Adm Saúde. 2007 Out-Dez; 9(37): 133-140.

24. D'innocenzo M, Cunha NPA, Olm ICK. O movimento pela qualidade nos serviços de saúde e enfermagem. Rev Bras Enf. 2006 Jan-Fev; 59(1): 84-88.

ISSN 1695-6141

@) COPYRIGHT Servicio de Publicaciones - Universidad de Murcia 